\title{
Aplicación de calceína para la estimación del crecimiento de la almeja amarilla Mesodesma mactroides Reeve, 1854
}

\author{
Application of calcein for estimating growth of the yellow clam \\ Mesodesma mactroides Reeve, 1854 \\ Mauro L. Lepore ${ }^{1}$, Pablo E. Penchaszadeh ${ }^{1}$, José E. F. Alfaya ${ }^{1}$ y Marko Herrmann² \\ ${ }^{1}$ Lab. Invertebrados, Depto. de Biodiversidad y Biología Experimental, Facultad de Ciencias Exactas y Naturales, Universidad \\ de Buenos Aires, Ciudad Universitaria, Pabellón II, $4^{\circ}$ piso, Lab. 19, C1428EHA, Buenos Aires, Argentina \\ ${ }^{2}$ Alfred Wegener Institute for Polar and Marine Research (AWI), Section of Marine Animal Ecology, \\ Postfach 120161, D-27515, Bremerhaven, Germany \\ maurolepore@gmail.com
}

\begin{abstract}
Growth rates of Mesodesma mactroides were estimated comparing two different methods at the intertidal of the exposed sandy beach Santa Teresita, Argentina. Results of a short tagging-recapture experiment using the in situ fluorescent marking (MFI) method and subsequent sizeincrement analysis were compared with results from lengthfrequency distributions (DFT) analysis from a 25 month quantitative sampling. Residuals, derived from $M F I$ method
\end{abstract}

\section{Introducción}

La tasa de crecimiento de un organismo provee información ecológica básica e importante para el estudio de su dinámica poblacional. En los bivalvos, dado que muchas especies son importantes en la industria pesquera, este parámetro se ha estudiado detalladamente mediante numerosos métodos, entre ellos, análisis de distribuciones de frecuencia de tallas, análisis de anillos de crecimiento de la concha y análisis de incrementos de talla siguiendo experimentos de marcaje-recaptura. Las estimaciones de crecimiento y longevidad resultantes de métodos diferentes son a veces contradictorias, por ejemplo Capezzani et al. (1971) reportaron una longevidad de 8 años para Mesodesma mactroides Reeve, 1854, mientras que Defeo et al. (1988) sugirieron 3,5 años para la misma especie. Los métodos actuales para la determinación del crecimiento y la edad de los bivalvos tienen sus limitaciones específicas. Los análisis de distribuciones de frecuencia de tallas $(D F T)$ requieren cohortes de edad bien definida y, normalmente, gran tamaño muestral. La cuantificación de anillos de crecimiento de la concha es afectada por la erosión superficial y los eventos disruptivos (para revisiones de métodos de crecimiento ver Richardson 2001), mientras que los métodos de marcaje-recaptura invasivos promueven disturbios físicos $\mathrm{y}$, eventualmente, tasas de crecimiento no-características. and DFT analysis, were of similar magnitude and distribution, suggesting that both methods are equally appropriate to estimate growth of M. mactroides. Calcein was useful as non-lethal growth marker for $M$. mactroides, emitting a bright green fluorescence band under blue light.

Key words: In situ fluorescent marking, comparison of methods, daily growth rate, length-frequency distribution, overall growth performance index

Diferentes estudios han probado varios químicos como marcadores del crecimiento de la concha en diferentes invertebrados marinos (Nakahara 1961, Monaghan 1993, Pricker \& Schie 1993, Day et al. 1995, Peck et al. 1996); dentro de la diversidad de marcadores, se ha demostrado que la calceína es apropiada para investigar los incrementos de crecimiento en bivalvos después del marcaje (Kaehler \& McQuaid 1999, Fujikura et al. 2003, Heilmayer et al. 2005, Riascos et al. 2006, Riascos et al. 2008, Herrmann et al. 2009). La calceína polianiónica es un compuesto fluorescente que se une con el carbonato de calcio en estructuras en crecimiento biomineralizadas (como las conchas) de organismos y que emite un color verde lima fluorescente cuando se ve bajo luz azul (Wilson et al. 1987).

El objetivo del presente trabajo fue determinar la tasa de crecimiento diario in situ de la almeja amarilla Mesodesma mactroides Reeve, $1854^{1}$ (Bivalvia: Mesodesmatidae) sobre la base a un experimento de marcaje fluorescente in situ (MFI). Además, estimar los parámetros de la ecuación de crecimiento de von

\footnotetext{
${ }^{1}$ En estudios previos, Deshayes 1854 se citó erróneamente como el autor de la especie $M$. mactroides. Sin embargo, la descripción no fue publicada por Deshayes sino por su colega Reeve, por lo tanto, el autor correspondiente de M. mactroides es Reeve 1854 (ver Deshayes 1854; Reeve 1854).
} 
Bertalanffy $(E C V B)$ para comparar los resultados con los parámetros de crecimiento estimados a partir de $D F T$, comparando los residuos y aplicando un índice de eficiencia del crecimiento ( $E C$, traducido del término en inglés 'overall growth performance' OGP).

\section{Material y métodos}

\section{Análisis de incrementos de talla}

\section{Muestreo, tinción y experimento de crecimiento in situ}

A fin de estudiar el crecimiento de Mesodesma mactroides por medio del método de $M F I$, se recolectaron 548 individuos vivos de esta especie de bivalvo, en febrero de 2007. Se obtuvo un amplio rango de longitud anterior-posterior de la concha (apSL: 7-65 mm) según lo disponible ese mes. Los individuos fueron obtenidos por excavación a mano de la playa arenosa de la zona intermareal expuesta Santa Teresita (provincia de Buenos Aires, Argentina: $\left.36^{\circ} 32^{\prime} \mathrm{S}, 56^{\circ} 40^{\prime} \mathrm{W}\right)$. Se midió la $a p S L$ de todos los especímenes utilizando un calibre vernier digital Mitutoyo ${ }^{\circledR}$ (modelo 500-161U) con una precisión de 0,01 mm. Los bivalvos se colocaron en tanques con agua de mar aireada circulante a temperatura ambiente; 411 especímenes se tiñeron con calceína (Sigma, CAS 1461-15-0; $50 \mathrm{mg} \mathrm{L}^{-1}$ por $3 \mathrm{~h}$ ) en un tanque oscuro, para evitar la desintegración lumínica del químico fluorescente. Adicionalmente, un grupo control notratado de 137 especímenes, asignado al azar, se mantuvo en un tanque similar. Luego de la inmersión, las almejas tratadas y control se mantuvieron in situ en cuatro jaulas experimentales, que constituyeron las réplicas, en la zona intermareal expuesta de Santa Teresita. Las jaulas cúbicas, de $40 \mathrm{~cm}$ de lado, estaban recubiertas internamente con una malla de nylon de $1 \mathrm{~mm}$ de trama a través de la cual podía atravesar el sedimento y las microalgas $(<50 \mu \mathrm{m}$ : Coscarón 1959). En la parte superior tenían un cierre plástico que facilitó el muestreo. Las jaulas fueron instaladas dentro del cinturón de $M$. mactroides, enterradas en la arena a una profundidad aproximada de $35 \mathrm{~cm}$ y separadas al menos $10 \mathrm{~m}$ entre sí. Cada una de ellas fue asegurada por medio de un cabo subterráneo a un ancla enterrada en la región supralitoral. Para proteger el experimento se realizó guardia durante los 63 días que duró el experimento; se realizaron muestreos semanales, en que se desenterraron las jaulas y se sumergieron en agua de mar hasta lograr escurrir todo su contenido de arena. Luego se tomaron cinco organismos de cada jaula con cuidado suficiente para evitar dañar las conchas. Diariamente, se registraron y extrajeron de las jaulas experimentales los animales muertos que se visualizaban en la superficie del sedimento.

\section{Preparación de las conchas y detección de la tasa absoluta de crecimiento}

Los bivalvos muestreados se sacrificaron y sus conchas vacías se limpiaron y secaron a temperatura ambiente por 48 horas. Para la detección de las marcas incorporadas, producidas durante la inmersión, las conchas se incluyeron en resina Epoxicure (Distraltec LY 554 y HL 554) y se cortaron secciones transversales de la concha a lo largo del eje de mayor crecimiento mediante el uso de una sierra diamantada Buehler ${ }^{\circledR}$ (modelo Isomet). Posteriormente, los cortes resultantes se pulieron sobre planchas de vidrio con polvos Buehler ${ }^{\circledR}$ de carburo de silicio (SiC) de 500, 800 y 1000 phi. Las marcas de calceína se detectaron bajo un microscopio de fluorescencia (Zeiss ${ }^{\circledR}$ Axio Imager Z1) irradiado con luz azul (450 a $490 \mathrm{~nm}$ ) por medio de fotografías y examen mediante el uso del programa de procesamiento de imágenes digitales AxioVision (2008) versión 4.6.3.

La tasa absoluta de crecimiento de Mesodesma mactroides se midió como el crecimiento de la concha a través del tiempo:

Tasa absoluta de crecimiento $=\left(u m S L_{2}-u m S L_{1}\right) /$

$$
\left(t_{2}-t_{1}\right)=\Delta u m S L / \Delta t \quad(\text { Ec. } 1)
$$

donde, $u m S L_{1}$ es la longitud inicial de la concha (mm) entre el umbo y el margen de la concha antes de la tinción $\left(t_{1}\right)$, y $u m S L_{2}$ la longitud final de la concha $(\mathrm{mm})$ entre el umbo y el margen de la concha al final del período experimental $\left(t_{2}\right)$.

\section{Análisis de distribuciones de frecuencia de tallas}

\section{Muestreo y obtención de datos}

En la playa arenosa de Santa Teresita, donde se realizó el experimento in situ, se recolectaron mensualmente muestras cuantitativas de Mesodesma mactroides de una serie de estaciones (a intervalos de $4 \mathrm{~m}$ ), desde diciembre de 2004 a diciembre de 2006. Las estaciones de muestreo estaban ubicadas a lo largo de tres transectos separados 20 m entre sí, y dispuestos perpendicularmente a la línea de costa desde la marca de marea alta de sizigia hasta la marca de marea baja de sizigia. En cada estación se excavaron tres muestras de arena replicadas $(40 \mathrm{x} 40 \mathrm{~cm})$ a $35 \mathrm{~cm}$ de profundidad mediante el uso de una parcela (cubo sin tapa ni fondo) de acero. Luego, las muestras se tamizaron individualmente en una malla de $1 \mathrm{~mm}$ de trama y la apSL de las almejas retenidas se midió utilizando un calibre vernier digital Mitutoyo ${ }^{\circledR}$ (modelo 500-161U) con una precisión de $0,01 \mathrm{~mm}$ para obtener las $D F T$ mensuales. 


\section{Comparación de métodos}

A fin de evaluar la conveniencia del experimento de marcaje-recaptura mediante el uso del método de marcaje fluorescente in situ en comparación con el método de análisis de distribuciones de frecuencia de tallas, se compararon las estimaciones del crecimiento del método de MFI con las del método de análisis de DFT. Los datos de $D F T$ se interpretaron como datos de talla-a-edad (DTE). El conjunto de datos de $M F I$ constituido por valores de $u m S L$ se convirtieron a datos de apSL por la ecuación de regresión lineal $u m S L=0,771 \cdot a p S L+0,914$ $\left(N=44, r^{2}=0,996\right)$. A los datos de incrementos de talla (DIT) resultantes del método de $M F I$ y a los DTE resultantes del análisis de $D F T$, se les ajustaron ecuaciones de crecimiento de von Bertalanffy generales (ECVBgs) mediante el uso de la planilla de cálculo computacional de $\mathrm{Brey}^{2}$, aplicando la rutina SOLVER de Microsoft Office Excel (2007):

$$
L_{t}=L_{\infty}\left(1-\mathrm{e}^{-K[t-t 0]}\right)^{\mathrm{D}}
$$

donde $L_{t}$ es la $\operatorname{apSL}(\mathrm{mm})$ a tiempo $t, L_{\infty}$ es la $\operatorname{apS} L(\mathrm{~mm})$ asintótica media, $K$ la constante de crecimiento $\left(\mathrm{añno}^{-1}\right)$, $D$ determina la forma de la curva (punto de inflexión si $D>1)$, y $t_{0}$ es la edad cuando la apSL iguala a cero.

Asimismo, se calculó el índice $E C$, que se utilizó para comparar la $E C V B$ s de diferentes locaciones geográficas. Varios autores (e.g. Pauly 1979, Munro \& Pauly 1983, Moreau et al. 1986, Laudien et al. 2003, Defeo \& Cardoso 2004) demostraron que los índices compuestos de EC son apropiados para comparaciones inter $\mathrm{e}$ intraespecíficas. La $E C$ es proporcional a la máxima tasa de incremento de masa corporal a lo largo del tiempo de vida, o sea, el incremento de masa en el punto de inflexión de la $E C V B$, dado que son pocos los valores de máxima masa corporal que se pueden hallar en la literatura y que la masa máxima es proporcional a $L_{\infty}$. En este contexto, la $E C$ de $M$. mactroides derivada de ambos métodos usados en este estudio se calculó de la siguiente manera:

$$
E C=\log \left(K\left[L_{\infty}\right]^{3}\right) \quad \text { (Ec. 3) }
$$

y se comparó con resultados de varias poblaciones de Mesodesma de diferentes áreas.

${ }^{2}$ Brey T. 2001. Population dynamics in benthic invertebrates. A virtual handbook. Version 01.2. Alfred Wegener Institute for Polar and Marine Research, Germany. [en línea] <http:// www.thomas-brey.de/science/virtualhandbook>

\section{Análisis estadístico}

Se realizó un análisis de Chi-cuadrado $\left(\chi^{2}\right)$ para determinar si la tinción de las almejas produjo diferencias significativas en las tasas de mortalidad durante el experimento de crecimiento in situ. La relación entre la $u m S L_{2}$ y la tasa de crecimiento diario se estimó en base al análisis de regresión exponencial. Los efectos de la $u m S L_{2}$ y el tiempo de exposición sobre la tasa de crecimiento se analizaron mediante un ANCOVA de una vía (tasa de crecimiento como variable dependiente, días de exposición como factores fijos y largo inicial como covariante). Los experimentos de análisis de DFT y de marcaje-recaptura por el método de $M F I$ y el subsiguiente análisis de incrementos de talla usados para estimar el crecimiento de ambos grupos de almejas se compararon por medio de un ANOVA de los residuos (diferencia entre la muestra y el valor de la función de ajuste) de las ECVBgs. Todos los análisis estadísticos se llevaron a cabo mediante el uso del paquete estadístico SPSS (2007) versión 16.0.1. Las diferencias estadísticas entre las variables y grupos a comparar se consideraron significativas a un nivel de $\alpha \leq 5 \%$ (Zar 1999).

\section{Resultados}

\section{Análisis de incrementos de talla}

Las jaulas descritas demostraron ser adecuadas para el experimento de $M F I$ en la zona intermareal expuesta. Todas las jaulas resistieron la exposición a las olas durante todo el período experimental. Visualmente, no se determinaron diferencias en la turbidez del agua dentro y fuera de las jaulas, no se reconocieron residuos filtrados en la malla ni se registraron taponamientos por sedimento en la malla, lo cual indica condiciones de alimentación naturales para los especímenes testeados.

El marcador fluorescente 'calceína' bajo luz azul emitió una banda verde brillante de fluorescencia, la cual fue claramente distinguida de la autofluorescencia de ocurrencia natural, a la concentración y el tiempo de exposición utilizados. Las marcas de calceína fueron conspicuas en el $83 \%(\mathrm{~N}=81)$ de los especímenes observados, dentro de los cuales se hallaron y midieron incrementos de crecimiento en el $94 \%(\mathrm{~N}=76)$. La mortalidad fue entre $14 \%(\mathrm{~N}=71)$ y $23 \%(\mathrm{~N}=117)$ para los especímenes teñidos, y de $18 \%(\mathrm{~N}=93)$ en la jaula control. Por lo tanto, el marcaje con calceína no afectó la supervivencia de Mesodesma mactroides $\left(\chi^{2}=0,736, d f\right.$ $=3, P=0,865) \mathrm{y}$, entonces, la calceína es un marcador no-letal útil para experimentos de campo.

Como se esperaba, los máximos incrementos de crecimiento se registraron en los individuos más pequeños 


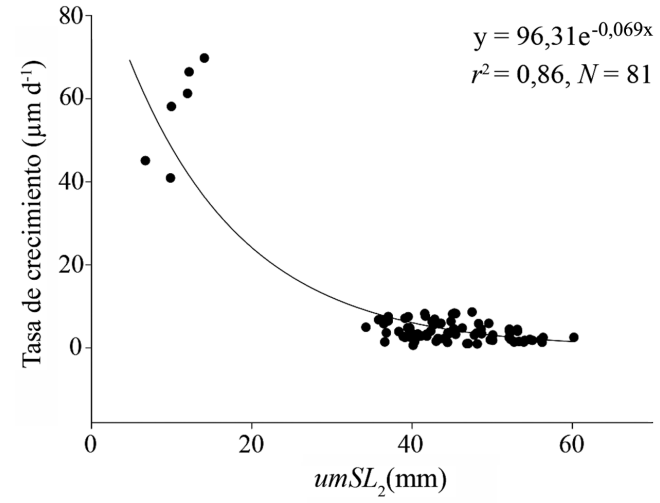

Figura 1

M. mactroides. Relación entre longitud final de la concha entre el umbo y el margen de la concha $\left(u m S L_{2}\right)$ $y$ tasa de crecimiento $\left(\mu \mathrm{m} \mathrm{d}^{-1}\right)$

M. mactroides. Relationship between final shell length between umbo and shell margin $\left(u m S L_{2}\right)$ and growth rate $\left(\mu \mathrm{m} \mathrm{d}^{-1}\right)$

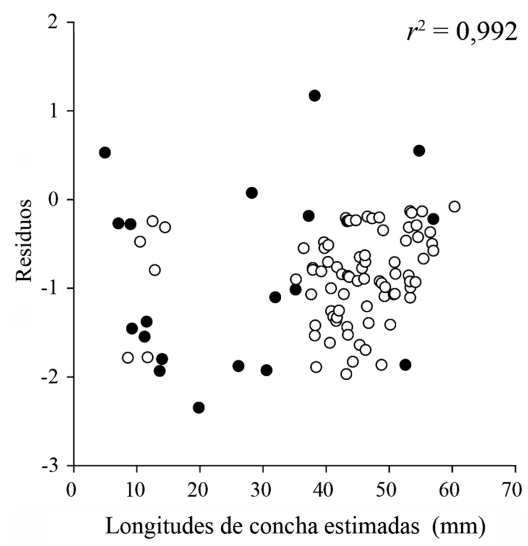

Figura 2

M. mactroides. Residuos de longitudes de concha estimadas de los datos de talla-a-edad (DTE) convertidos del método de distribuciones de frecuencia de tallas $(D F T)(\bullet)$ y de los datos de incrementos de talla (DIT) del método de marcaje fluorescente in situ (MFI) (O), mostrando diferencia no significativa (ANOVA,

$$
\left.F_{1,98}=0,019, P>0,05\right)
$$

M. mactroides. Residuals of estimated shell lengths of size-atage data $(D T E)$ converted from length-frequency distributions $(D F T)$ method $(\bullet)$ and of size-increment data $(D I T)$ from in situ fluorescent marking (MFI) method (0), showing no significant difference (ANOVA,

$$
\left.F_{1,98}=0.019, P>0.05\right)
$$

de $M$. mactroides (por ejemplo, $u m S L_{2}=8,70 \mathrm{~mm}+3,13$ $\mathrm{mm}$ en 45 días). La tasa de crecimiento diario individual osciló entre $0,46 \mu \mathrm{m} \mathrm{d}^{-1}$ y $69,6 \mu \mathrm{m} \mathrm{d}^{-1}$. La relación entre $u m S L_{2}$ y la tasa de crecimiento diario fue descrita mejor por una función exponencial (Fig. 1). La longitud inicial de la concha en el experimento $\left(F_{1,70}=51,35, P<0,05\right)$ tuvo efectos significativos en la tasa de crecimiento de las almejas, y el tiempo de exposición $\left(F_{9,70}=1,87, P>\right.$ $0,05)$ tuvo efectos no significativos en la tasa de crecimiento de las almejas (ANCOVA). El crecimiento disminuyó exponencialmente de las almejas pequeñas a las más grandes $\left(\mathrm{y}=96,31 \mathrm{e}^{-0,069 \mathrm{x}}, r^{2}=0,86, \mathrm{~N}=81\right)$ (Fig. 1).

Se ajustó una $E C V B g$ a los DIT, originados del $M F I$, mediante el uso de la talla máxima $(u m S L=55 \mathrm{~mm}$ [correspondiente a $a p S L=71 \mathrm{~mm}$ ]) obtenida por el método de $D F T$ como valor fijo de $L_{\infty}$ para calcular la constante de crecimiento $K=0,48$ año $^{-1}\left(r^{2}=0,39\right)$.

\section{Análisis de distribuciones de frecuencia de tallas}

Durante 25 meses se recolectaron y midieron 3.015 ejemplares de Mesodesma mactroides en Santa Teresita (primer año $\mathrm{N}=2.142$ ind., segundo año $\mathrm{N}=873$ ind.). El individuo más pequeño registrado tuvo una $a p S L$ de 2 mm y el más grande midió 64 mm (apSL). Los parámetros de crecimiento de la $E C V B$ estimada para la población de $M$. mactroides de Santa Teresita fueron $K=0,47\left(\right.$ año $^{-1}$ ) y $L_{\infty}=71 \mathrm{~mm}($ apSL).

\section{Comparación de métodos}

Los resultados obtenidos mediante el método de $M F I$ se compararon con los resultados de $D F T$ por medio del análisis de los residuos de las ECVBgs. La gráfica de residuos en comparación con las longitudes de concha estimadas mostró un ajuste alto $\left(r^{2}=0,99\right)$ (Fig. 2). El análisis de varianza de los residuos de las $E C V B g$ s mostró una diferencia no significativa entre los dos métodos (ANOVA, $\left.F_{1,98}=0,019, P>0,05\right)$.

Los valores de EC computados de Mesodesma mactroides que resultan del $M F I(E C=5,24)$ y las $D F T$ $(E C=5,23)$ son próximos entre sí al ser graficados dentro de la grilla auximétrica, que compara la $E C$ de varias poblaciones de Mesodesma de distintas áreas (Fig. 3, $\mathrm{N}^{\circ}$ 1 y $\mathrm{N}^{\circ} 2$, respectivamente).

\section{Discusión}

Este estudio ha demostrado que la aplicación de la calceína es útil para la estimación del crecimiento de la almeja amarilla argentina. La eficiencia en la marcación de Mesodesma mactroides de esta investigación concuerda con observaciones previas que afirman que la 
calceína produce marcas claras en moluscos bajo condiciones controladas, lo que hace posible estudios de crecimiento de corta duración y alta resolución (e.g. Haliotis rubra: Day et al. 1995, Perna perna: Kaehler \& McQuaid 1999, Adamussium colbecki: Heilmayer et al. 2005, Concholepas concholepas y Mesodesma donacium: Riascos et al. 2006). Los primeros estudios de crecimiento mediante el uso de fluorocromos demostraron que los organismos eran adversamente afectados y sufrían una mortalidad substancialmente distinta a la natural (Schmitt 1984, Monaghan 1993, Day et al. 1995). Luego, otros trabajos probaron una variedad de fluorocromos y mostraron que la calceína presentaba poca toxicidad (Wilson et al. 1987, Hales Jr \& Hurley 1991, Monaghan 1993, Day et al. 1995, Rowley \& Mackinnon 1995). En concordancia con estudios recientes (Morán 2000, Riascos et al. 2006, Herrmann et al. 2009), la presente investigación reveló que el marcaje con calceína no afectó la supervivencia de $M$. mactroides durante el experimento in situ. Esto respalda que la calceína pueda ser recomendada como marcador no-letal para $M$. mactroides y otros bivalvos.

La banda fluorescente distintiva y angosta incorporada en el borde en crecimiento de la concha en el momento de la exposición a la calceína se utilizó exitosamente como punto de partida en las mediciones del crecimiento. Las marcas fluorescentes se detectaron con certeza en muestras almacenadas al menos 15 meses después del experimento sin degradación notable de las marcas de crecimiento. Por lo tanto, el potencial del uso de la calceína como marcador del crecimiento en estudios a largo plazo es muy grande (véase también Rowley \& Mackinnon 1995, Kaehler \& McQuaid 1999, Morán 2000, Riascos et al. 2006, Herrmann et al. 2009).

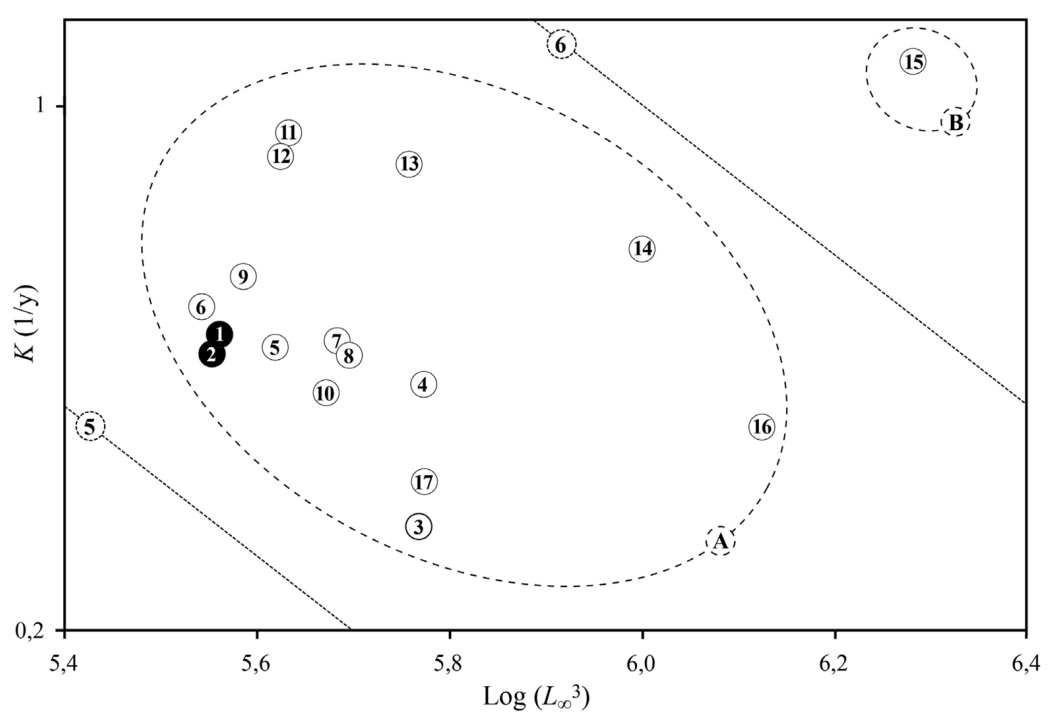

Figura 3

Grilla auximétrica comparando la eficiencia del crecimiento $(E C)$ (Ec. 3) de M. mactroides argentina calculada del método de marcaje fluorescente in situ (MFI) (1) y del método de distribuciones de frecuencia de tallas (DFT) (2) del presente estudio

(•), con varias poblaciones de Mesodesma de diferentes áreas de investigaciones previas (O). Se indican dos grupos: regiones templadas (A) y área de surgencia (B). Las líneas diagonales punteadas unen puntos de igual valor de

EC (números en círculos punteados). Fuente de datos: M. mactroides de Argentina (3: Capezzani et al. 1971, 5-10: Fiori \& Morsán 2004, 4: Luzzatto 2007), M. mactroides de Uruguay (13, 14: Defeo et al. 1992a, 11, 12: Defeo et al. 1992b), M. donacium de Perú (15-17: Arntz et al. 1987)

Auximetric grid comparing the overall growth performance $(E C)$ (Ec. 3) of the Argentinean M. mactroides calculated from in situ fluorescent marking (MFI) method (1) and from the length-frequency distributions (DFT) method (2) from the present study (•), with several Mesodesma populations from different areas from previous research (0).Two groups are indicated: temperate (A) and upwelling (B) zones. Dotted diagonal lines indicate equal values of $E C$ (numbers in dotted circles). Data sources: $M$. mactroides from Argentina (3: Capezzani et al. 1971, 5-10: Fiori \& Morsán 2004, 4: Luzzatto 2007), M. mactroides from Uruguay (13, 14: Defeo et al. 1992a, 11, 12: Defeo et al. 1992b), 
El experimento in situ permitió registrar en los especímenes de Mesodesma mactroides un crecimiento mínimo distinguible de $0,46 \mu \mathrm{m} \mathrm{d}^{-1} \mathrm{y}$ un crecimiento máximo de $69,6 \mu \mathrm{m} \mathrm{d}^{-1}$, estando la tasa de crecimiento diario correlacionada con la $u m S L_{2}$ como lo describe una función exponencial, dependiente de la talla de los individuos (Fig. 1).

Los residuos, derivados del método de MFI y del análisis de $D F T$, fueron de magnitud y distribución similares. El crecimiento de las almejas amarillas calculado en el experimento in situ de 63 días por MFI, concuerda bien con la observación de 25 meses del crecimiento de las conchas por análisis de DFT. Más aún, los valores de $E C$ de Mesodesma mactroides resultantes del MFI y DFT de esta investigación, muestran una pequeña variación con respecto a los valores calculados a partir de otros conjuntos de datos de la misma especie en distintas locaciones de la costa argentina: Por ejemplo el punto $\mathrm{N}^{\circ} 3$ de la Fig. 3 corresponde al área de Faro Querandí (37 $\left.29^{\prime} \mathrm{S}, 5^{\circ} 07^{\prime} \mathrm{W}\right)$, los puntos $\mathrm{N}^{\circ}$ 5-10 fueron determinados para las áreas de Monte Hermoso (38 $59^{\circ} \mathrm{S}$, $\left.61^{\circ} 15^{\prime} \mathrm{W}\right)$ e Isla Jabalí (403' $\left.\mathrm{S}, 62^{\circ} 14^{\prime} \mathrm{W}\right)$, y el punto $\mathrm{N}^{\circ} 4$ fue determinado para el área de Costa Chica $\left(36^{\circ} 31^{\prime} \mathrm{S}, 56^{\circ} 41^{\prime} \mathrm{W}\right)$, todas locaciones de la provincia de Buenos Aires. Por lo tanto se sugiere que alternativamente a los análisis de $D F T$, los experimentos de marcajerecaptura usando el método de $M F I$ y subsiguiente análisis de incrementos de talla son apropiados para estimar el crecimiento de la almeja amarilla argentina M. mactroides.

La grilla auximétrica (Fig. 3) que compara la $E C$ de varias poblaciones de Mesodesma sugiere también que los experimentos de marcaje-recaptura mediante el uso del método de MFI y subsiguiente análisis de incrementos de talla son requeridos para estimar el crecimiento de especies tropicales, dado que ellas exhiben eventos reproductivos o de reclutamiento continuos a lo largo de períodos mas prolongados, comparado con los Mesodesma de áreas templadas, por lo que resulta difícil el seguimiento de las cohortes y entonces el análisis de DFT por sí solo podría no ser útil para estimar el crecimiento de las especies tropicales (Sparre \& Venema 1998). En tal sentido, los experimentos de marcajerecaptura asociados por ejemplo, al método de $M F I$, son recomendados para estimar adecuadamente el crecimiento de bivalvos tropicales.

Sin embargo, el método de $M F I$ y el de $D F T$ tienen ventajas y desventajas. Un gran punto a favor del $M F I$ es la precisión, ya que permite determinar la de tasa de crecimiento diario de $M$. mactroides a partir de mediciones directas. Además, requiere de un número de especímenes relativamente bajo en comparación con el análisis de DFT. Por lo tanto, el MFI se puede utilizar en poblaciones esparcidas en las que resulta difícil muestrear suficientes especímenes para la detección clara de cohortes. Por otra parte, el análisis de DFT permite la detección de crecimiento estacional (Appeldoorn 1987) y evita el sacrificio de especímenes para la obtención de datos. Aunque el presente estudio mostró parámetros de crecimiento calculados a partir de MFI similares a los de $D F T$, es posible que el primer método no siempre detecte el crecimiento de los adultos con precisión, debido a la lenta tasa de crecimiento y el corto período de tiempo experimental. Por lo tanto, el MFI se debe aplicar durante un período adecuado, dependiente de la tasa de crecimiento de la especie. En contraste, los largos períodos de estudio necesarios para el análisis de DFT son vulnerables al sesgo causado por la migración de las cohortes a lo largo de la costa, embancamiento y posibles mortandades masivas, dificultad en la localización del banco por dinámica costera y formación de parches y distribución discontinua en la línea de playa. Adicionalmente, llevar a cabo solo experimentos de corta duración podría conducir a una subestimación de $L_{\infty}$ debido a la posible falta de individuos adultos grandes. En ese caso, los análisis de DFT son más precisos debido al período de muestreo más prolongado. Además, la exposición a olas fuertes puede destruir un experimento in situ por completo, mientras que la recolección de datos del análisis de DFT podría ser pospuesta. Finalmente, el método de $M F I$ requiere una mano de obra mas esforzada y un equipamiento más costoso; A pesar de ello, considerando que el microscopio de fluorescencia puede alquilarse o solicitarse en préstamo, el estudio del crecimiento por $M F I$ resulta ser mas económico que por análisis de $D F T$.

\section{Agradecimientos}

Este trabajo es parte de la tesis de licenciatura de Mauro Lepore, financiada en parte por el 'COA Grants to Malacology'. Los autores agradecen a Atilio Mita, Diego Canton, Maximiliano Jakavasky y otros compañeros guardavidas que colaboraron durante el experimento en la playa; a Diana Avellaneda cuya ayuda con los cortes fue imprescindible; a los compañeros de laboratorio por su hospitalidad y consejos; a la revisora Valeria Climenti y a los evaluadores.

\section{Literatura citada}

Appeldoorn R. 1987. Modification of a seasonally oscillating growth function for use with mark-recapture data. Journal du Conseil Permanent International pour l'Exploration de la Mer 43: 194-198. 
Arntz WE, T Brey, J Tarazona \& A Robles. 1987. Changes in the structure of a shallow sandy-beach community in Peru during an El Niño event. South African Journal of Marine Science 5: 645-658.

AxioVision. 2008. Digital Image Processing Software, Carl Zeiss MicroImaging GmbH. [CD-ROM]

Capezzani DAA, SR Oliver \& PE Penchaszadeh. 1971. Dinámica de población. En: Oliver SR, DAA Capezzani, J Carreto, H Christiansen, V Moreno, JA de Moreno \& PE Penchaszadeh (eds). Estructura de la comunidad, dinámica de la población y biología de la almeja amarilla (Mesodesma mactroides Desh. 1854) en Mar Azul (Pdo. de Gral. Madariaga, Bs.As., Argentina). Proyecto de Desarrollo Pesquero FAO/PNUD. Serie Informes Técnicos 27: 49-64.

Coscarón S. 1959. La almeja amarilla (Mesodesma (T.) mactroides Deshayes) de la costa de la Provincia de Buenos Aires. Dirección de Agricultura e Industrias: Publicaciones Técnicas 1(3): 1-66.

Day RW, MC Williams \& GP Hawkes. 1995. A comparison of fluorochromes for marking abalone shells. Marine and Freshwater Research 46: 599-605.

Defeo O \& RS Cardoso. 2004. Latitudinal patterns in abundance and life-history traits of the mole crab Emerita brasiliensis on South American sandy beaches. Diversity and Distributions 10: 89-98.

Defeo O, F Arreguín-Sanchez \& J Sánchez. 1992a. Growth study of the yellow clam Mesodesma mactroides: a comparative analysis of three length-based methods. Scientia Marina 56(1): 53-59.

Defeo O, A Masello \& C Layerle. 1988. Consideraciones metodológicas para el estudio del crecimiento en moluscos bivalvos. Informe Unesco Ciencias del Mar 47: 135-148.

Defeo O, E Ortiz \& JC Castilla. 1992b. Growth, mortality and recruitment of the yellow clam Mesodesma mactroides on Uruguayan beaches. Marine Biology 114: 429-437.

Deshayes MGP. 1854. Descriptions of new shells from the collection of Hugh Cuming, Esq. Proceedings of the Zoological Society of London 22: 317-371.

Fiori SM \& EM Morsán. 2004. Age and individual growth of Mesodesma mactroides (Bivalvia) in the southernmost range of its distribution. Journal of Marine Science 61: 1253-1259.

Fujikura K, K Okoshi \& T Naganuma. 2003. Strontium as a marker for estimation of microscopic growth rates in a bivalve. Marine Ecology Progress Series 257: 295-301.

Hales LS Jr \& DH Hurley. 1991. Validation of daily increment formation in the otoliths of juvenile silver perch, Bairdiella chrysoura. Estuaries 14: 199-206.

Heilmayer O, C Honnen, U Jacob, M Chiantore, R CattaneoVietti \& T Brey. 2005. Temperature effects on summer growth rates in the Antarctic scallop, Adamussium colbecki. Polar Biology 28: 523-527.
Herrmann M, J Laudien, PE Penchaszadeh \& WE Arntz. 2009. Growth estimations of the Argentinean wedge clam Donax hanleyanus: A comparison between lengthfrequency distribution and size-increment analysis. Journal of Experimental Marine Biology and Ecology 379: 8-15.

Kaehler S \& CD McQuaid. 1999. Use of the fluorochrome calcein as an in situ growth marker in the brown mussel Perna perna. Marine Biology 133: 455-460.

Laudien J, T Brey \& WE Arntz. 2003. Population structure, growth and production of the surf clam Donax serra (Bivalvia, Donacidae) on two Namibian sandy beaches. Estuarine, Coastal and Shelf Science 58S: 105-115.

Luzzatto DC. 2007. Dinámica poblacional de la almeja amarilla (Mesodesma mactroides) y del berberecho (Donax hanleyanus) en intermareales de playas arenosas del Nordeste de la Provincia de Buenos Aires. Tesis doctoral, Facultad de Ciencias Exactas y Naturales, Universidad de Buenos Aires, Buenos Aires, 186 pp.

Monaghan JP. 1993. Comparison of calcein and tetracycline as chemical markers in summer flounder. Transactions of the American Fisheries Society 122: 298-301.

Morán AL. 2000. Calcein as a marker in experimental studies newly-hatched gastropods. Marine Biology 137: 893-898.

Moreau J, C Bambino \& D Pauly. 1986. Indices of overall growth performance of 100 tilapia (Cichlidae) populations. En: Maclean JL, LB Dizon \& LV Hosillos (eds). The first Asian fisheries forum, pp. 201-206. Asian Fisheries Society, Manila.

Munro JL \& D Pauly. 1983. A simple method for comparing the growth of fishes and invertebrates. Fishbyte 1: 5-6.

Nakahara H. 1961. Determination of calcein and tetracycline as chemical markers in summer flounder. Bulletin of the National Pearl Research Laboratory 6: 607-614.

Pauly D. 1979. Gill size and temperature as governing factors in fish growth: a generalization of the von Bertalanffy's growth formula. Berichte aus dem Institut für Meereskunde an der Christian-Albrechts-Universität Kiel 63: 1-156.

Peck LS, AC Baker \& LZ Conway. 1996. Strontium labelling of the shell of the Antarctic limpet Nacella concinna (Strebel 1908). Journal of Molluscan Studies 62: 315-325.

Pricker JG \& DR Schie. 1993. Tetracycline as a fluorescent shell-marker in the abalone Haliotis iris. Marine Biology: 81-86.

Reeve LA. 1854. Monograph of the genus Mesodesma. Conchologia Iconica 8: 1-4.

Riascos JM, N Guzman, J Laudien, O Heilmayer \& M Oliva. 2006. Suitability of three stains to mark shells of Concholepas concholepas (Gastropopda) and Mesodesma donacium (Bivalvia). Journal of Shellfish Research 26(1): $1-7$.

Riascos JM, O Heilmayer, ME Oliva, J Laudien \& WE Arntz. 2008. Infestation of the surf clam Mesodesma 
donacium by the spionid polychaete Polydora bioccipitalis. Journal of Sea Research 59: 217-227.

Richardson CA. 2001. Molluscs as archives of environmental change. Oceanography and Marine Biology: an Annual Review 39: 103-164.

Rowley RJ \& DI Mackinnon. 1995. Use of the fluorescent marker calcein in biomineralisation studies of brachiopods and other marine organisms. Bulletin de l'Institut Océanographique, Monaco 14(part 2): 111-120.

Schmitt PD. 1984. Marking growth increments in otoliths of larval and juvenile fish by immersion in tetracycline to examine the rate of increment formation. Fishery Bulletin 82: $237-242$.
Sparre P \& SC Venema. 1998. Introduction to tropical fish stock assessment. Part 1. Manual. FAO Fisheries Technical Paper 306/1 Rev. 2: 1-440.

SPSS. 2007. Statistical Package for the Social Sciences, Version 16.0.1 SPSS inc. Chicago, Illinois.[CD-ROM]

Wilson CW, DW Beckman \& JM Dean. 1987. Calcein as a fluorescent marker of otoliths of larval and juvenile fish. Transactions of the American Fisheries Society 116: 668670.

Zar JH. 1999. Biostatistical analysis, 663 pp. Prentice-Hall, Upper Saddle River.

Recibido el 8 de febrero de 2009 y aceptado el 14 de julio de 2009 